Article

\title{
Edge-Version Atom-Bond Connectivity and Geometric Arithmetic Indices of Generalized Bridge Molecular Graphs
}

\author{
Xiujun Zhang ${ }^{1 \oplus}$, Xinling $\mathrm{Wu}^{2}$, Shehnaz Akhter ${ }^{3}$, Muhammad Kamran Jamil ${ }^{4}$, Jia-Bao Liu ${ }^{5, * \mathbb{C}}$ \\ and Mohammad Reza Farahani ${ }^{6}$ (D) \\ 1 Key Laboratory of Pattern Recognition and Intelligent Information Processing, Institutions of Higher \\ Education of Sichuan Province, Chengdu University, Chengdu 610106, China; woodszhang@cdu.edu.cn \\ 2 South China Business College, Guang Dong University of Foreign Studies, Guangzhou 510545, China; \\ xinlingwu.guangzhou@gmail.com \\ 3 Department of Mathematics, School of Natural Sciences (SNS), National University of Sciences and \\ Technology (NUST), Sector H-12, Islamabad 44000, Pakistan; shehnazakhter36@yahoo.com \\ 4 Department of Mathematics, Riphah Institute of Computing and Applied Sciences, Riphah International \\ University Lahore, Lahore 54660, Pakistan; m.kamran.sms@gmail.com \\ 5 School of Mathematics and Physics, Anhui Jianzhu University, Hefei 230601, China \\ 6 Department of Applied Mathematics, Iran University of Science and Technology, Narmak, \\ Tehran 16844, Iran; mrfarahani88@gmail.com \\ * Correspondence: liujiabao@ahjzu.edu.cn
}

Received: 29 November 2018; Accepted: 13 December 2018; Published: 14 December 2018

\begin{abstract}
Topological indices are graph invariants computed by the distance or degree of vertices of the molecular graph. In chemical graph theory, topological indices have been successfully used in describing the structures and predicting certain physicochemical properties of chemical compounds. In this paper, we propose a definition of generalized bridge molecular graphs that can model more kinds of long chain polymerization products than the bridge molecular graphs, and provide some results of the edge versions of atom-bond connectivity $\left(A B C_{e}\right)$ and geometric arithmetic $\left(G A_{e}\right)$ indices for some generalized bridge molecular graphs, which have regular, periodic and symmetrical structures. The results of this paper offer promising prospects in the applications for chemical and material engineering, especially in chemical industry research.
\end{abstract}

Keywords: atom-bond connectivity index; geometric arithmetic index; line graph; generalized bridge molecular graph

\section{Introduction}

Let $G$ be an undirected simple graph without loops or multiple edges. We denote by $V(G)$ the vertex set of $G$ and we denote by $E(G)$ the edge set of $G$. We denote by $e=u v$ the edge connect vertices $u$ and $v$ or vertices $u$ and $v$ adjacent. We denote by $P_{n}, C_{n}$, and $S_{n}$ the path, cycle, and star of $n$ vertices, respectively. We denote by $N(v)$ the open neighborhood of vertex $v$, i.e., $N(v)=\{u \mid u v \in E(G)\}$. We denote by $d(v)$ or $d_{G}(v)$ the degree of a vertex $v$ of a graph $G$, i.e., $d(v)=|\{u \in N(v)\}|$. Let $L(G)$ or $G^{L}$ be a line graph of $G$, so each vertex of $L(G)$ corresponds an edge of $G$. Two vertices of $L(G)$ are adjacent if and only if a common endpoint is shared by their corresponding edges in $G$ [1]. The degree of edge $e$ in $G$ is denoted by $d_{L(G)}(e)$, which is the number of edges that share common endpoint with edge $e$ in $G$; it is also the degree of vertex $e$ in $L(G)$. We give simple a illustration to explain the relationship of original graph and corresponding line graph in Figure 1. We can see $u, v, w$ denote corresponding vertexes, and $e, f, g, h, i, j$ denote corresponding edges in original graph $G$ and 
denote corresponding vertices in line graph $L(G)$. We get $d(u)=d(v)=3, d(w)=2$. $d_{L(G)}(e)$, which is the degree of vertex $e$ in $L(G)$, is also the degree of edge $e$ in $G$, thus $d_{L(G)}(e)=4$ in Figure 1 .

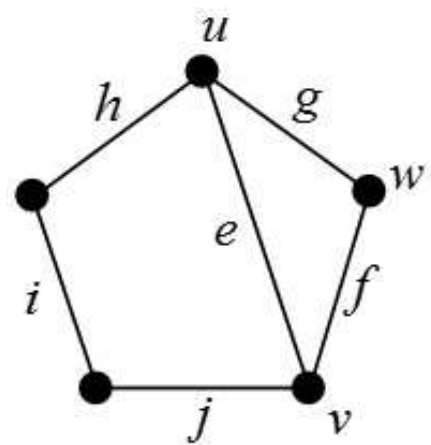

$G$

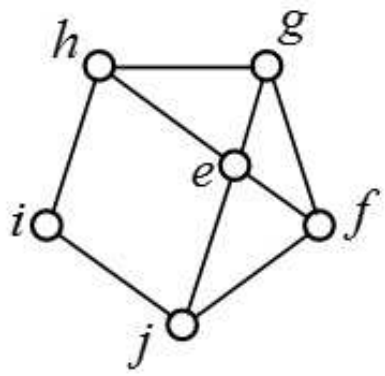

$L(G)$

Figure 1. The original graph $G$ and corresponding line graph $L(G)$.

Topological indices are graph invariants, which are obtained by performing some numerical operations on the distance or degree of vertices of the molecular graph. In chemical graph theory, topological indices are the molecular descriptors. They have been successfully used in describing the structures and predicting certain physicochemical properties of chemical compounds. To study the relationship between molecular structure and physical properties of saturated hydrocarbons, Wiener index was first published in 1947 [2], and the edge version of Wiener index, which can be considered as the Wiener index of line graph of $G$, was proposed by Iranmanesh et al. in 2009 [3]. As the important role of topological indices in chemical research has been confirmed, more topological indices appeared, which include atom-bond connectivity index and geometric arithmetic index.

In chemical graph theory, hydrogen atoms are usually ignored when the topological indices are calculated, which is very similar to how organic chemists usually simply write a benzene ring as a hexagon [4]. Now, three types of graphs of $\mathrm{C}_{24} \mathrm{H}_{28}$ are illustrated in Figure 2.

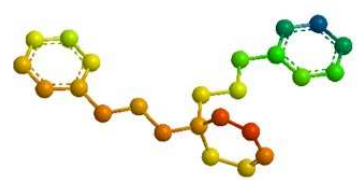

(a)

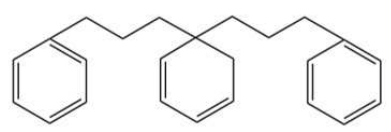

(b)

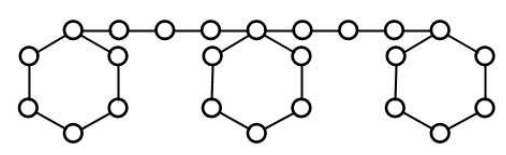

(c)

Figure 2. (a) $\mathrm{C}_{24} \mathrm{H}_{28}$ ball and stick model graph in $3 D$; (b) $\mathrm{C}_{24} \mathrm{H}_{28}$ chemical structure graph; and (c) $\mathrm{C}_{24} \mathrm{H}_{28}$ model graph in chemical graph theory.

To explore the properties of simple short chain compound products, Gao et al. [5] defined some join graphs such as $P_{n}+C_{m}, P_{n}+S_{m}, C_{m}+P_{n}+C_{m}, S_{m}+P_{n}+S_{m}$, and $C_{m}+P_{n}+S_{r}$, created by $P_{n}, C_{n}$ and $S_{n}$ and obtained the $A B C_{e}$ and $G A_{e}$ indices of these graphs. In another paper, Gao et al. [6] defined the bridge molecular structures, which can be used to research some long chain polymerization products, and the forgotten indices $(F(G))$ formulae of some simple bridge molecular structures constructed by $P_{2}, C_{6}$ or $K_{3}$ are presented. The forgotten index is defined as $F(G)=\sum_{v \in V(G)}\left(d(v)^{3}\right)[7]$. In this paper, we define generalized bridge molecular graphs that could cover more kinds of long chain polymerization products, and the edge-version atom-bond connectivity and geometric arithmetic indices of generalized bridge molecular graphs are calculated.

To facilitate the reader, the topological indices discussed in this thesis are all given in Table 1. 
Table 1. The definition of topological indices.

\begin{tabular}{llcc}
\hline Index Name & Definition & Proposed & Recent Studied \\
\hline atom-bond connection index & $A B C(G)=\sum_{u v \in E(G)} \sqrt{\frac{d(u)+d(v)-2}{d(u) d(v)}}$ & {$[8]$} & {$[9-11]$} \\
\hline edge version of $A B C$ index & $A B C_{e}(G)=\sum_{e_{1} e_{2} \in E(L(G))} \sqrt{\frac{d_{L(G)}\left(e_{1}\right)+d_{L(G)}\left(e_{2}\right)-2}{d_{L(G)}\left(e_{1}\right) \times d_{L(G)}\left(e_{2}\right)}}$ & {$[12]$} & {$[5,13,14]$} \\
\hline geometric arithmetic index & $G A(G)=\sum_{u v \in E(G)} \frac{2 \sqrt{d_{G}(u) d_{G}(v)}}{d_{G}(u)+d_{G}(v)}$ & {$[15]$} & {$[16-18]$} \\
\hline edge version of GA index & $G A_{e}(G)=\sum_{e_{1} e_{2} \in E(L(G))} \frac{2 \sqrt{d_{L(G)}\left(e_{1}\right) d_{L(G)}\left(e_{2}\right)}}{d_{L(G)}\left(e_{1}\right)+d_{L(G)}\left(e_{2}\right)}$ & {$[19]$} & {$[5,12,19-21]$} \\
\hline
\end{tabular}

In Table $1, d_{G}(u)$ and $d_{G}(v)$ are the degrees of the vertices $u$ and $v$ in $G$, and $d_{L(G)}\left(e_{1}\right)$ and $d_{L(G)}\left(e_{2}\right)$ are the degrees of the edges $e_{1}$ and $e_{2}$ in $G$.

\section{Main Results and Proofs}

\subsection{Definition of the Generalized Bridge Molecular Graph}

Before we start a discussion, we give the definition of the generalized bridge molecular graph as follows. For a positive integer $d, d$ pairwise disjoint molecular graphs $\left\{G^{(1)}, G^{(2)}, \ldots, G^{(d)}\right\}$ with $v^{(i)} \in$ $V\left(G^{(i)}\right)$ for each $i=1,2, \cdots, d$, and $d-1$ pairwise disjoint path molecular graphs $P^{(1)}, P^{(2)}, \ldots, P^{(d-1)}$ (called bridges), the generalized bridge molecular graph $G B G\left(G^{(1)}, v^{(1)}, G^{(2)}, v^{(2)}, \ldots, G^{(d)}, v^{(d)} ; P^{(1)}\right.$, $\left.P^{(2)}, \ldots, P^{(d-1)}\right)$ is the graph obtained by connecting the vertices $v^{(i)}$ and $v^{(i+1)}$ by a path $P^{(i)}$ for which two end vertices are identified with $v^{(i)}$ and $v^{(i+1)}$ for $i=1,2, \ldots, d-1$ (See Figure 3). When $G:=G^{(i)}, P:=P^{(i)}, v:=v^{(i)}$ for each $i$, we simplify $G B G\left(G^{(1)}, v^{(1)}, G^{(2)}, v^{(2)}, \cdots, G^{(d)}, v^{(d)} ; P^{(1)}\right.$, $\left.P^{(2)}, \ldots, P^{(d-1)}\right)$ to be $G B G(G, v ; P ; d)$. In this paper, if $G$ is a star, then $v$ is the central vertex and if $G$ is a cycle, $v$ is considered as any vertex. In such cases, we further simplify $G B G(G, v ; P ; d)$ to be $G B G(G, P ; d)$. The bridge molecular graph's bridge is strictly $P_{2}$ in $[6]$, which limits the scope of modeling objects. The generalized bridge molecular graphs can model more kinds of long chain polymerization products than the bridge molecular graphs, because the bridge can be either $P_{2}$ or $P_{n}$ and $n \geq 3$.

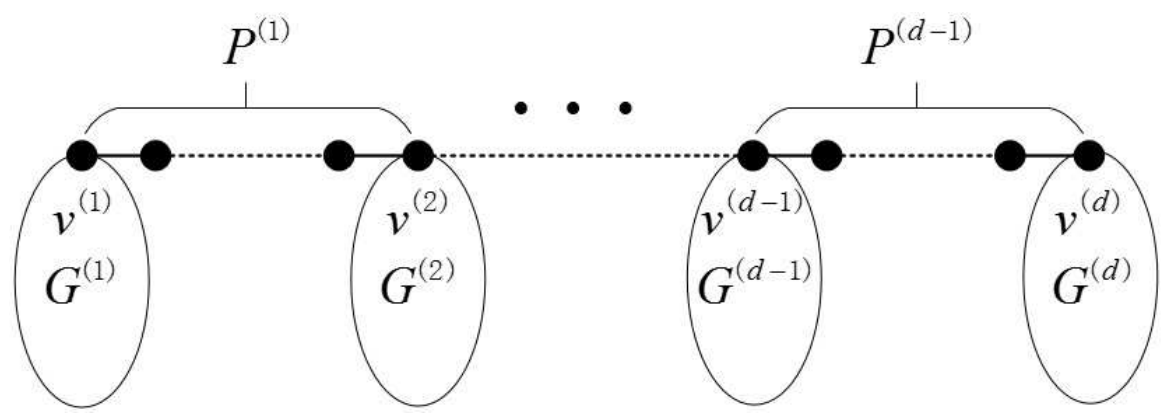

Figure 3. The generalized bridge molecular graph $G B G\left(G^{(1)}, v^{(1)}, G^{(2)}, v^{(2)}, \ldots, G^{(d)}, v^{(d)} ; P^{(1)}, P^{(2)}, \ldots, P^{(d-1)}\right)$.

\subsection{Results and Discussion}

In the following, we discuss the edge-version atom-bond connectivity and geometric arithmetic indices of some generalized bridge molecular graph. The line graph $G B G^{L}\left(S_{m}, P_{n} ; d\right)$ of $G B G\left(S_{m}, P_{n} ; d\right)$ is illustrated in Figure 4. 

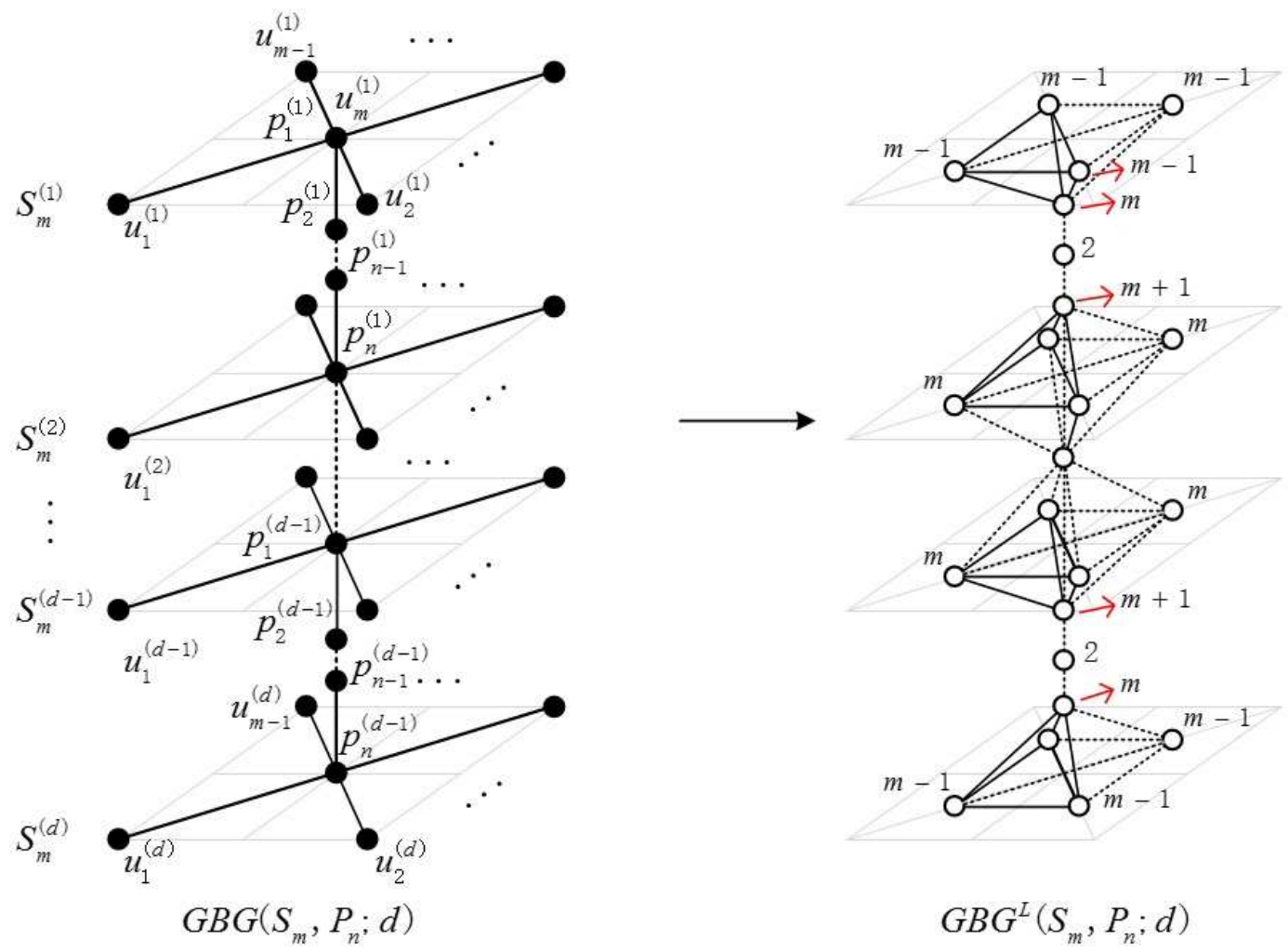

Figure 4. The generalized bridge molecular graph of $G B G\left(S_{m}, P_{n} ; d\right)$ and $G B G^{L}\left(S_{m}, P_{n} ; d\right)$.

Theorem 1. Let $G B G\left(S_{m}, P_{n} ; d\right)$ be the generalized bridge molecular graph for $n \geq 4, d \geq 2$ and $m \geq 2$ (see Figure 4), then the $A B C_{e}$ and $G A_{e}$ of $G B G\left(S_{m}, P_{n} ; d\right)$ are

$$
\begin{aligned}
A B C_{e}\left(G B G\left(S_{m}, P_{n} ; d\right)\right)= & \frac{\sqrt{2}}{2}(d-1)(n-2)+\frac{d-2}{m+1} \sqrt{2 m} \\
& +2(m-1) \sqrt{\frac{2 m-3}{m^{2}-m}}+2(d-2)(m-1) \sqrt{\frac{2 m-1}{m^{2}+m}} \\
& +(m-2) \sqrt{2 m-4}+\frac{(d-2)(m-1)(m-2) \sqrt{2 m-2}}{2 m}, \\
G A_{e}\left(G B G\left(S_{m}, P_{n} ; d\right)\right)= & \frac{4 \sqrt{2 m}}{m+2}+\frac{4(d-2) \sqrt{2 m+2}}{m+3}+(d-1)(n-4)+(d-2) \\
& +4(m-1) \frac{\sqrt{m^{2}-m}}{2 m-1}+4(d-2)(m-1) \frac{\sqrt{m^{2}+m}}{2 m+1} \\
& +\frac{d}{2}(m-1)(m-2) .
\end{aligned}
$$

Proof. This line graph has $2-2 m-n+\frac{d}{2}\left(m^{2}+m+2 n-4\right)$ edges. If $d_{L(G)}\left(e_{1}\right)$ and $d_{L(G)}\left(e_{2}\right)$ are the degree of edge of $e_{1}$ and $e_{2}$, then there are 2 edges of type $d_{L(G)}\left(e_{1}\right)=m, d_{L(G)}\left(e_{2}\right)=2,2(d-2)$ edges of type $d_{L(G)}\left(e_{1}\right)=m+1, d_{L(G)}\left(e_{2}\right)=2,(d-1)(n-4)$ edges of type $d_{L(G)}\left(e_{1}\right)=d_{L(G)}\left(e_{2}\right)=2, d-2$ edges of type $d_{L(G)}\left(e_{1}\right)=d_{L(G)}\left(e_{2}\right)=m+1,2(m-1)$ edges of type $d_{L(G)}\left(e_{1}\right)=m, d_{L(G)}\left(e_{2}\right)=m-1$, $2(d-2)(m-1)$ edges of type $d_{L(G)}\left(e_{1}\right)=m, d_{L(G)}\left(e_{2}\right)=m+1,(m-1)(m-2)$ edges of type $d_{L(G)}\left(e_{1}\right)=d_{L(G)}\left(e_{2}\right)=m-1$, and $\frac{d-2}{2}(m-1)(m-2)$ edges of type $d_{L(G)}\left(e_{1}\right)=d_{L(G)}\left(e_{2}\right)=m$. Hence, we get 


$$
\begin{aligned}
& A B C_{e}\left(G B G\left(S_{m}, P_{n} ; d\right)\right)=2\left(\sqrt{\frac{m+2-2}{m \times 2}}\right)+2(d-2)\left(\sqrt{\frac{m+1+2-2}{(m+1) \times 2}}\right) \\
& +(d-1)(n-4)\left(\sqrt{\frac{2+2-2}{2 \times 2}}\right) \\
& +(d-2)\left(\sqrt{\frac{m+1+m+1-2}{(m+1) \times(m+1)}}\right) \\
& +2(m-1)\left(\sqrt{\frac{m+m-1-2}{m \times(m-1)}}\right) \\
& +2(d-2)(m-1)\left(\sqrt{\frac{m+m+1-2}{m \times(m+1)}}\right) \\
& +(m-1)(m-2)\left(\sqrt{\frac{m-1+m-1-2}{(m-1) \times(m-1)}}\right) \\
& +\frac{d-2}{2}(m-1)(m-2)\left(\sqrt{\frac{m+m-2}{m \times m}}\right) \\
& =\frac{\sqrt{2}}{2}(d-1)(n-2)+\frac{d-2}{m+1} \sqrt{2 m} \\
& +2(m-1) \sqrt{\frac{2 m-3}{m^{2}-m}}+2(d-2)(m-1) \sqrt{\frac{2 m-1}{m^{2}+m}} \\
& +(m-2) \sqrt{2 m-4}+\frac{(d-2)(m-1)(m-2) \sqrt{2 m-2}}{2 m}, \\
& G A_{\mathcal{e}}\left(G B G\left(S_{m}, P_{n} ; d\right)\right)=2\left(\frac{2 \sqrt{m \times 2}}{m+2}\right)+2(d-2)\left(\frac{2 \sqrt{(m+1) \times 2}}{m+1+2}\right) \\
& +(d-1)(n-4)\left(\frac{2 \sqrt{2 \times 2}}{2+2}\right) \\
& +(d-2)\left(\frac{2 \sqrt{(m+1) \times(m+1)}}{m+1+m+1}\right) \\
& +2(m-1)\left(\frac{2 \sqrt{m \times(m-1)}}{m+m-1}\right) \\
& +2(d-2)(m-1)\left(\frac{2 \sqrt{m \times(m+1)}}{m+m+1}\right) \\
& +(m-1)(m-2)\left(\frac{2 \sqrt{(m-1) \times(m-1)}}{m-1+m-1}\right) \\
& +\frac{d-2}{2}(m-1)(m-2)\left(\frac{2 \sqrt{m \times m}}{m+m}\right) \\
& =\frac{4 \sqrt{2 m}}{m+2}+\frac{4(d-2) \sqrt{2 m+2}}{m+3}+(d-1)(n-4)+(d-2) \\
& +4(m-1) \frac{\sqrt{m^{2}-m}}{2 m-1}+4(d-2)(m-1) \frac{\sqrt{m^{2}+m}}{2 m+1} \\
& +\frac{d}{2}(m-1)(m-2) \text {. }
\end{aligned}
$$

The proof is complete. 
For Example 1, in Figure 5, 2,7,7,12 - tetramethyltridecane can be modeled by $G B G\left(S_{3}, P_{6} ; 3\right)$, so $A B C_{e}\left(G B G\left(S_{3}, P_{6} ; 3\right)\right) \approx 13.76052$ and $G A_{e}\left(G B G\left(S_{3}, P_{6} ; 3\right)\right) \approx 19.72337$.

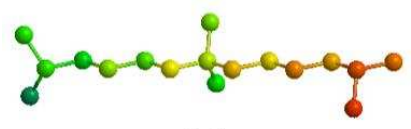

(a)

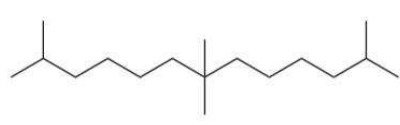

(b)

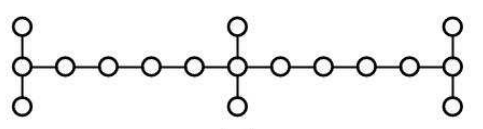

(c)

Figure 5. (a) 2,7,7,12-tetramethyltridecane ball and stick model graph in 3D; (b) 2,7,7,12-tetramethyltridecane chemical structure graph; and (c) 2,7,7,12-tetramethyltridecane model graph in chemical graph theory.

Theorem 2. Let $G B G\left(S_{m}, P_{3} ; d\right)$ be the generalized bridge molecular graph for $n=3, d \geq 3$ and $m \geq 2$ (see Figure 6), then the $A B C_{e}$ and $G A_{e}$ of $G B G\left(S_{m}, P_{3} ; d\right)$ are

$$
\begin{aligned}
A B C_{e}\left(G B G\left(S_{m}, P_{3} ; d\right)\right)= & 2 \sqrt{\frac{2 m-1}{m^{2}+m}}+\frac{2 d-5}{m+1} \sqrt{2 m}+2(m-1) \sqrt{\frac{2 m-3}{m^{2}-m}} \\
+ & 2(d-2)(m-1) \sqrt{\frac{2 m-1}{m^{2}+m}}+(m-2) \sqrt{2 m-4} \\
+ & \frac{(d-2)(m-1)(m-2)}{2 m} \sqrt{2 m-2}, \\
G A_{e}\left(G B G\left(S_{m}, P_{3} ; d\right)\right)= & \frac{4}{2 m+1} \sqrt{m^{2}+m}+(2 d-5) \\
& +\frac{4}{2 m-1}(m-1) \sqrt{m^{2}-m} \\
& +\frac{4}{2 m+1}(d-2)(m-1) \sqrt{m^{2}+m} \\
& +\frac{d}{2}(m-1)(m-2) .
\end{aligned}
$$
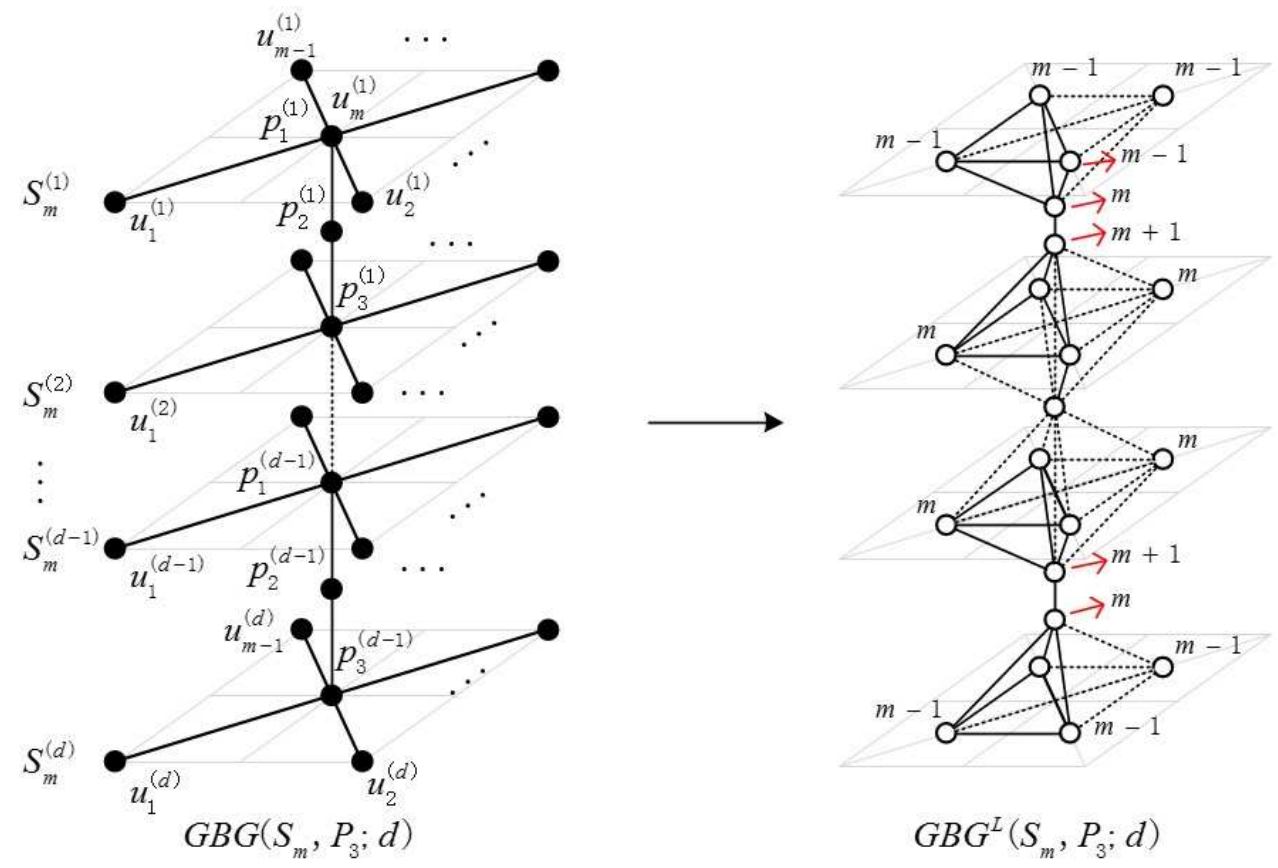

Figure 6. The generalized bridge molecular graph of $G B G\left(S_{m}, P_{3} ; d\right)$ and $G B G^{L}\left(S_{m}, P_{3} ; d\right)$. 
Proof. This line graph has $\frac{d}{2}\left(m^{2}+m+2\right)-2 m-1$ edges. If $d_{L(G)}\left(e_{1}\right)$ and $d_{L(G)}\left(e_{2}\right)$ are the degree of edge of $e_{1}$ and $e_{2}$, then there are 2 edges of type $d_{L(G)}\left(e_{1}\right)=m, d_{L(G)}\left(e_{2}\right)=m+1,2 d-5$ edges of type $d_{L(G)}\left(e_{1}\right)=m+1, d_{L(G)}\left(e_{2}\right)=m+1,2(m-1)$ edges of type $d_{L(G)}\left(e_{1}\right)=m, d_{L(G)}\left(e_{2}\right)=$ $m-1,2(d-2)(m-1)$ edges of type $d_{L(G)}\left(e_{1}\right)=m, d_{L(G)}\left(e_{2}\right)=m+1,(m-1)(m-2)$ edges of type $d_{L(G)}\left(e_{1}\right)=d_{L(G)}\left(e_{2}\right)=m-1$, and $\frac{d-2}{2}(m-1)(m-2)$ edges of type $d_{L(G)}\left(e_{1}\right)=d_{L(G)}\left(e_{2}\right)=m$. Hence, we get

$$
\begin{aligned}
& A B C_{e}\left(G B G\left(S_{m}, P_{3} ; d\right)\right)=2\left(\sqrt{\frac{m+m+1-2}{m \times(m+1)}}\right)+(2 d-5)\left(\sqrt{\frac{m+1+m+1-2}{(m+1) \times(m+1)}}\right) \\
& +2(m-1)\left(\sqrt{\frac{m+m-1-2}{m \times(m-1)}}\right) \\
& +2(d-2)(m-1)\left(\sqrt{\frac{m+m+1-2}{m \times(m+1)}}\right) \\
& +(m-1)(m-2)\left(\sqrt{\frac{m-1+m-1-2}{(m-1) \times(m-1)}}\right) \\
& +\frac{d-2}{2}(m-1)(m-2)\left(\sqrt{\frac{m+m-2}{m \times m}}\right) \\
& =2 \sqrt{\frac{2 m-1}{m^{2}+m}}+\frac{2 d-5}{m+1} \sqrt{2 m}+2(m-1) \sqrt{\frac{2 m-3}{m^{2}-m}} \\
& +2(d-2)(m-1) \sqrt{\frac{2 m-1}{m^{2}+m}}+(m-2) \sqrt{2 m-4} \\
& +\frac{(d-2)(m-1)(m-2)}{2 m} \sqrt{2 m-2} \text {, } \\
& G A_{e}\left(G B G\left(S_{m}, P_{3} ; d\right)\right)=2\left(\frac{2 \sqrt{m \times(m+1)}}{m+m+1}\right)+(2 d-5)\left(\frac{2 \sqrt{(m+1) \times(m+1)}}{m+1+m+1}\right) \\
& +2(m-1)\left(\frac{2 \sqrt{m \times(m-1)}}{m+m-1}\right) \\
& +2(d-2)(m-1)\left(\frac{2 \sqrt{m \times(m+1)}}{m+m+1}\right) \\
& +(m-1)(m-2)\left(\frac{2 \sqrt{(m-1) \times(m-1)}}{m-1+m-1}\right) \\
& +\frac{d-2}{2}(m-1)(m-2)\left(\frac{2 \sqrt{m \times m}}{m+m}\right) \\
& =\frac{4}{2 m+1} \sqrt{m^{2}+m}+(2 d-5) \\
& +\frac{4}{2 m-1}(m-1) \sqrt{m^{2}-m} \\
& +\frac{4}{2 m+1}(d-2)(m-1) \sqrt{m^{2}+m} \\
& +\frac{d}{2}(m-1)(m-2) \text {. }
\end{aligned}
$$

The proof is complete.

For Example 2, in Figure 7, 2,4,4,6 - tetramethylheptane can be modeled by $G B G\left(S_{3}, P_{3} ; 3\right)$, so $A B C_{e}\left(G B G\left(S_{3}, P_{3} ; 3\right)\right) \approx 9.394663$ and $G A_{e}\left(G B G\left(S_{3}, P_{3} ; 3\right)\right) \approx 13.85764$. 


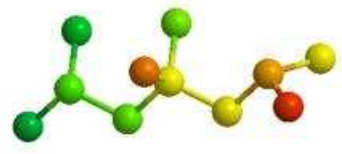

(a)

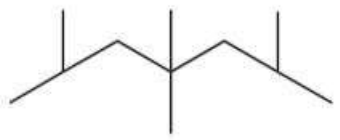

(b)

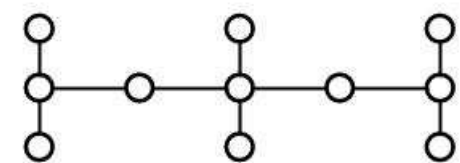

(c)

Figure 7. (a) 2, 4, 4, 6-tetramethylheptane ball and stick model graph in 3D; (b) 2,4,4,6-tetramethylheptane chemical structure graph; and (c) 2,4,4,6-tetramethylheptane model graph in chemical graph theory.

Theorem 3. Let $G B G\left(S_{m}, P_{2} ; d\right)$ be the generalized bridge molecular graph for $n=2, d \geq 4$ and $m \geq 2$ (see Figure 8), then the $A B C_{e}$ and $G A_{e}$ of $G B G\left(S_{m}, P_{2} ; d\right)$ are

$$
\begin{aligned}
A B C_{e}\left(G B G\left(S_{m}, P_{2} ; d\right)\right)= & 2(m-1) \sqrt{\frac{3 m-4}{2 m^{2}-3 m+1}}+2(m-1) \sqrt{\frac{3 m-3}{2 m^{2}-m}} \\
& +2 \frac{(d-3)(m-1)}{m} \sqrt{\frac{3 m-2}{2}}+(m-2) \sqrt{2 m-4} \\
& +\frac{(d-2)(m-1)(m-2)}{2 m} \sqrt{2 m-2} \\
& +2 \sqrt{\frac{4 m-3}{4 m^{2}-2 m}+\frac{d-4}{2 m} \sqrt{4 m-2},}
\end{aligned}
$$

$$
\begin{aligned}
G A_{e}\left(G B G\left(S_{m}, P_{2} ; d\right)\right)= & \frac{4(m-1)}{3 m-2} \sqrt{2 m^{2}-3 m+1}+\frac{4(m-1)}{3 m-1} \sqrt{2 m^{2}-m} \\
& +\frac{4 \sqrt{2}}{3}(d-3)(m-1)+\frac{d}{2}(m-1)(m-2) \\
& +\frac{4\left(\sqrt{4 m^{2}-2 m}\right)}{4 m-1}+(d-4) .
\end{aligned}
$$
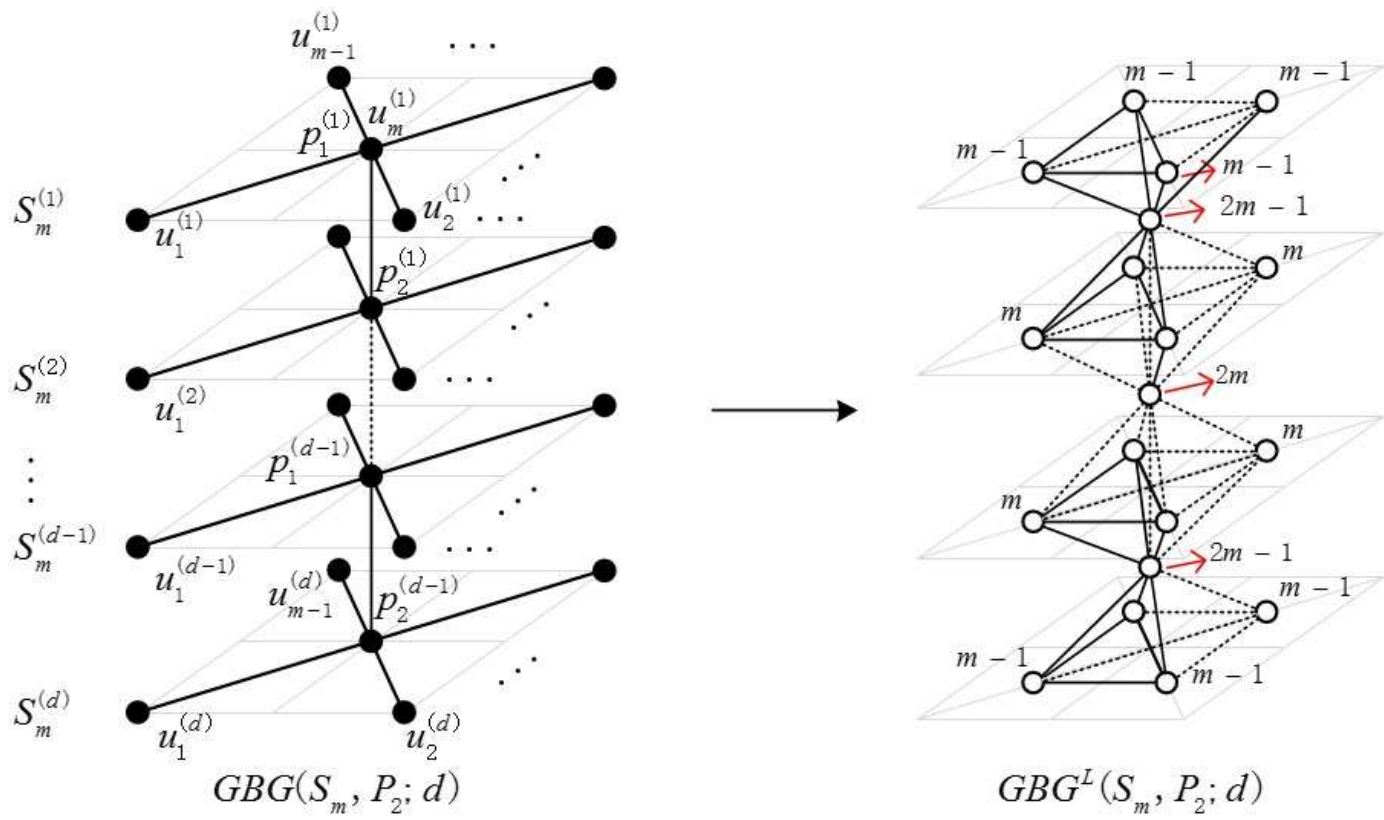

Figure 8. The generalized bridge molecular graph of $G B G\left(S_{m}, P_{2} ; d\right)$ and $G B G^{L}\left(S_{m}, P_{2} ; d\right)$.

Proof. This line graph has $\frac{1}{2} m(d m+d-4)$ edges. If $d_{L(G)}\left(e_{1}\right)$ and $d_{L(G)}\left(e_{2}\right)$ are the degree of edge of $e_{1}$ and $e_{2}$, then there are $2(m-1)$ edges of type $d_{L(G)}\left(e_{1}\right)=2 m-1, d_{L(G)}\left(e_{2}\right)=m-1,2(m-1)$ edges of 
type $d_{L(G)}\left(e_{1}\right)=2 m-1, d_{L(G)}\left(e_{2}\right)=m, 2(d-3)(m-1)$ edges of type $d_{L(G)}\left(e_{1}\right)=2 m, d_{L(G)}\left(e_{2}\right)=m$, $(m-1)(m-2)$ edges of type $d_{L(G)}\left(e_{1}\right)=d_{L(G)}\left(e_{2}\right)=m-1, \frac{d-2}{2}(m-1)(m-2)$ edges of type $d_{L(G)}\left(e_{1}\right)=d_{L(G)}\left(e_{2}\right)=m, 2$ edges of type $d_{L(G)}\left(e_{1}\right)=2 m-1, d_{L(G)}\left(e_{2}\right)=2 m$, and $d-4$ edges of type $d_{L(G)}\left(e_{1}\right)=d_{L(G)}\left(e_{2}\right)=2 m$. Hence, we get

$$
\begin{aligned}
& A B C_{e}\left(G B G\left(S_{m}, P_{2} ; d\right)\right)=2(m-1)\left(\sqrt{\frac{2 m-1+m-1-2}{(2 m-1) \times(m-1)}}\right) \\
& +2(m-1)\left(\sqrt{\frac{2 m-1+m-2}{(2 m-1) \times m}}\right) \\
& +2(d-3)(m-1)\left(\sqrt{\frac{2 m+m-2}{2 m \times m}}\right) \\
& +(m-1)(m-2)\left(\sqrt{\frac{m-1+m-1-2}{(m-1) \times(m-1)}}\right) \\
& +\frac{d-2}{2}(m-1)(m-2)\left(\sqrt{\frac{m+m-2}{m \times m}}\right) \\
& +2\left(\sqrt{\frac{2 m-1+2 m-2}{(2 m-1) \times 2 m}}\right) \\
& +(d-4)\left(\sqrt{\frac{2 m+2 m-2}{2 m \times 2 m}}\right) \\
& =2(m-1) \sqrt{\frac{3 m-4}{2 m^{2}-3 m+1}}+2(m-1) \sqrt{\frac{3 m-3}{2 m^{2}-m}} \\
& +2 \frac{(d-3)(m-1)}{m} \sqrt{\frac{3 m-2}{2}}+(m-2) \sqrt{2 m-4} \\
& +\frac{(d-2)(m-1)(m-2)}{2 m} \sqrt{2 m-2} \\
& +2 \sqrt{\frac{4 m-3}{4 m^{2}-2 m}}+\frac{d-4}{2 m} \sqrt{4 m-2}, \\
& G A_{e}\left(G B G\left(S_{m}, P_{2} ; d\right)\right)=2(m-1)\left(\frac{2 \sqrt{(2 m-1) \times(m-1)}}{2 m-1+m-1}\right) \\
& +2(m-1)\left(\frac{2 \sqrt{(2 m-1) \times m}}{2 m-1+m}\right) \\
& +2(d-3)(m-1)\left(\frac{2 \sqrt{2 m \times m}}{2 m+m}\right) \\
& +(m-1)(m-2)\left(\frac{2 \sqrt{(m-1) \times(m-1)}}{m-1+m-1}\right) \\
& +\frac{d-2}{2}(m-1)(m-2)\left(\frac{2 \sqrt{m \times m}}{m+m}\right) \\
& +2\left(\frac{2 \sqrt{(2 m-1) \times 2 m}}{2 m-1+2 m}\right) \\
& +(d-4)\left(\frac{2 \sqrt{2 m \times 2 m}}{2 m+2 m}\right) \\
& =\frac{4(m-1)}{3 m-2} \sqrt{2 m^{2}-3 m+1}+\frac{4(m-1)}{3 m-1} \sqrt{2 m^{2}-m} \\
& +\frac{4 \sqrt{2}}{3}(d-3)(m-1)+\frac{d}{2}(m-1)(m-2) \\
& +\frac{4\left(\sqrt{4 m^{2}-2 m}\right)}{4 m-1}+(d-4) \text {. }
\end{aligned}
$$


The proof is complete.

For Example 3, in Figure 9, 2,3,3,4-tetramethylpentane can be modeled by $G B G\left(S_{3}, P_{2} ; 4\right)$, so $A B C_{e}\left(G B G\left(S_{3}, P_{2} ; 4\right)\right) \approx 11.69568$ and $G A_{e}\left(G B G\left(S_{3}, P_{2} ; 4\right)\right) \approx 17.24996952$.

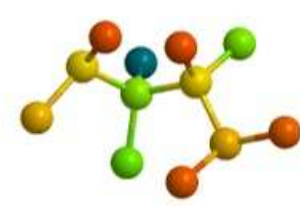

(a)

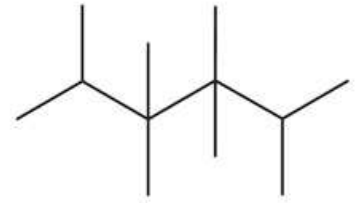

(b)

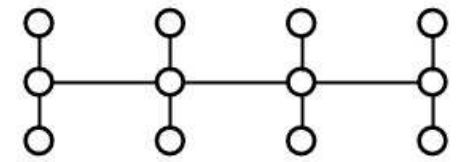

(c)

Figure 9. (a) 2,3,3,4-tetramethylpentane ball and stick model graph in 3D; (b) 2,3,3,4-tetramethylpentane chemical structure graph; and (c) 2,3,3,4-tetramethylpentane model graph in chemical graph theory.

Theorem 4. Let $G B G\left(C_{m}, P_{n} ; d\right)$ be the generalized bridge molecular graph for $n \geq 4, d \geq 2$ and $m \geq 3$ (see Figure 10), then the $A B C_{e}$ and $G A_{e}$ of $G B G\left(C_{m}, P_{n} ; d\right)$ are

$$
\begin{aligned}
A B C_{e}\left(G B G\left(C_{m}, P_{n} ; d\right)\right)= & \frac{\sqrt{2}}{2}(d(m-3)+(d-1)(n-4))+\left(2 \sqrt{2}+\frac{3 \sqrt{6}}{2}\right) d \\
-\sqrt{2}-3 \sqrt{6}+4 & \\
G A_{e}\left(G B G\left(C_{m}, P_{n} ; d\right)\right)= & d(m-3)+(d-1)(n-4) \\
& +\left(\frac{8 \sqrt{2}}{3}+6\right)(d-2)+\frac{12 \sqrt{6}}{5}+5 .
\end{aligned}
$$
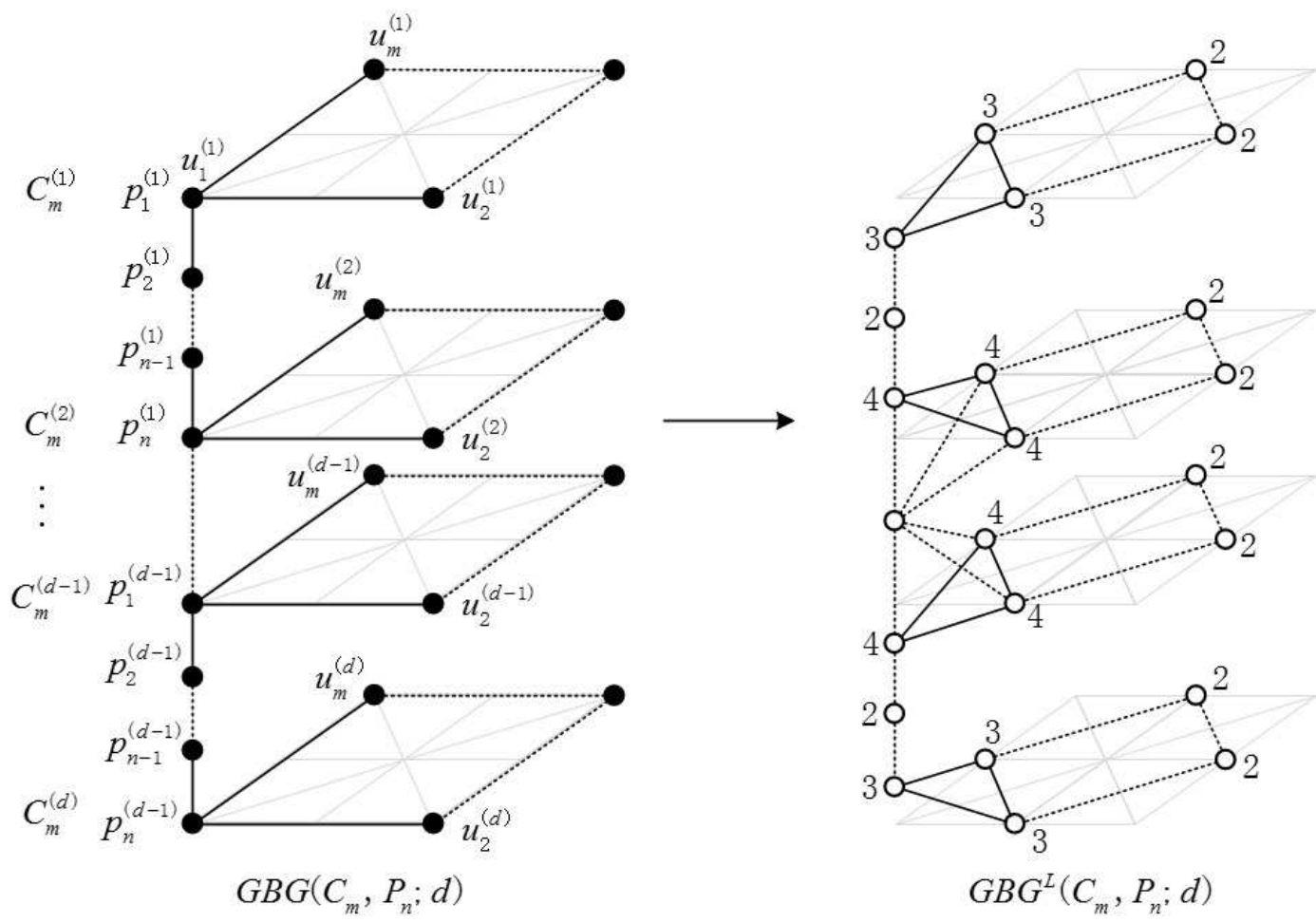

Figure 10. The generalized bridge molecular graph of $G B G\left(C_{m}, P_{n} ; d\right)$ and $G B G^{L}\left(C_{m}, P_{n} ; d\right)$. 
Proof. In Figure 10, the degrees of vertices in line graph $G^{L}\left(G_{d}\left(C_{m}+P_{n}\right)\right)$ are displayed near by the corresponding vertices. This line graph has $d(m+n+3)-n-4$ edges. In addition, there are $d(m-3)+(d-1)(n-4)$ edges of type $d_{L(G)}\left(e_{1}\right)=d_{L(G)}\left(e_{2}\right)=2,6$ edges of type $d_{L(G)}\left(e_{1}\right)=2$ and $d_{L(G)}\left(e_{2}\right)=3,6$ edges of type $d_{L(G)}\left(e_{1}\right)=d_{L(G)}\left(e_{2}\right)=3,4(d-2)$ edges of type $d_{L(G)}\left(e_{1}\right)=2$ and $d_{L(G)}\left(e_{2}\right)=4$, and $6(d-2)$ edges of type $d_{L(G)}\left(e_{1}\right)=d_{L(G)}\left(e_{2}\right)=4$. Hence, we have

$$
\begin{aligned}
A B C_{e}\left(G B G\left(C_{m}, P_{n} ; d\right)\right)= & (d(m-3)+(d-1)(n-4))\left(\sqrt{\frac{2+2-2}{2 \times 2}}\right) \\
& +6\left(\sqrt{\frac{2+3-2}{2 \times 3}}\right)+6\left(\sqrt{\frac{3+3-2}{3 \times 3}}\right) \\
& +4(d-2)\left(\sqrt{\frac{2+4-2}{2 \times 4}}\right)+6(d-2)\left(\sqrt{\frac{4+4-2}{4 \times 4}}\right) \\
= & \frac{\sqrt{2}}{2}(d(m-3)+(d-1)(n-4))+\left(2 \sqrt{2}+\frac{3 \sqrt{6}}{2}\right) d \\
- & \sqrt{2}-3 \sqrt{6}+4 \\
G A_{e}\left(G B G\left(C_{m}, P_{n} ; d\right)\right)= & (d(m-3)+(d-1)(n-4))\left(\frac{2 \sqrt{2 \times 2}}{2+2}\right) \\
& +6\left(\frac{2 \sqrt{2 \times 3}}{2+3}\right)+6\left(\frac{2 \sqrt{3 \times 3}}{3+3}\right) \\
& +4(d-2)\left(\frac{2 \sqrt{2 \times 4}}{2+4}\right)+6(d-2)\left(\frac{2 \sqrt{4 \times 4}}{4+4}\right) \\
= & d(m-3)+(d-1)(n-4) \\
& +\left(\frac{8 \sqrt{2}+6)(d-2)+\frac{12 \sqrt{6}}{5}+5 .}{3}\right.
\end{aligned}
$$

The proof is complete.

For Example 4, in Figure 2, $\mathrm{C}_{24} \mathrm{H}_{28}$ is (cyclohexa-2, 4-diene-1,1-diylbis(propane-3, 1-diyl))dibenzene, which can be modeled by $G B G\left(C_{6}, P_{5} ; 3\right)$, so $A B C_{e}\left(G B G\left(C_{6}, P_{5} ; 3\right)\right) \approx 22.52347702$ and $G A_{e}\left(G B G\left(C_{6}, P_{5} ; 3\right)\right) \approx 31.65001155$.

Theorem 5. Let $G B G\left(C_{m}, P_{3} ; d\right)$ be the generalized bridge molecular graph for $n=3, d \geq 3$, and $m \geq 3$ (see Figure 11), then the $A B C_{e}$ and $G A_{e}$ of $G B G\left(C_{m}, P_{3} ; d\right)$ are

$$
\begin{aligned}
& A B C_{e}\left(G B G\left(C_{m}, P_{3} ; d\right)\right)=\frac{\sqrt{2}}{2} d(m-3)+\left(\sqrt{2}+7 \frac{\sqrt{6}}{4}\right) d+4+\frac{\sqrt{15}}{3}-\frac{15 \sqrt{6}}{4}, \\
& G A_{e}\left(G B G\left(C_{m}, P_{3} ; d\right)\right)=d(m-3)+\left(\frac{4 \sqrt{2}}{3}+7\right) d+\frac{8 \sqrt{6}}{5}+6+\frac{8 \sqrt{3}}{7}-\frac{8 \sqrt{2}}{3}-15 .
\end{aligned}
$$

Proof. In Figure 11, the degrees of vertices in line graph $G^{L}\left(G B G\left(C_{m}, P_{3} ; d\right)\right)$ are displayed near by the corresponding vertices. This line graph has $d(m+6)-7$ edges. In addition, there are $d(m-3)$ edges of type $d_{L(G)}\left(e_{1}\right)=d_{L(G)}\left(e_{2}\right)=2,4$ edges of type $d_{L(G)}\left(e_{1}\right)=2$ and $d_{L(G)}\left(e_{2}\right)=3,2(d-2)$ edges of type $d_{L(G)}\left(e_{1}\right)=2$ and $d_{L(G)}\left(e_{2}\right)=4,6$ edges of type $d_{L(G)}\left(e_{1}\right)=d_{L(G)}\left(e_{2}\right)=3,2$ edges of type $d_{L(G)}\left(e_{1}\right)=3$ and $d_{L(G)}\left(e_{2}\right)=4$, and $7 d-15$ edges of type $d_{L(G)}\left(e_{1}\right)=d_{L(G)}\left(e_{2}\right)=4$. Hence, we have 


$$
\begin{aligned}
& A B C_{e}\left(G B G\left(C_{m}, P_{3} ; d\right)\right)=d(m-3)\left(\sqrt{\frac{2+2-2}{2 \times 2}}\right)+4\left(\sqrt{\frac{2+3-2}{2 \times 3}}\right) \\
& +2(d-2)\left(\sqrt{\frac{2+4-2}{2 \times 4}}\right)+6\left(\sqrt{\frac{3+3-2}{3 \times 3}}\right) \\
& +2\left(\sqrt{\frac{3+4-2}{3 \times 4}}\right)+(7 d-15)\left(\sqrt{\frac{4+4-2}{4 \times 4}}\right) \\
& =\frac{\sqrt{2}}{2} d(m-3)+\left(\sqrt{2}+7 \frac{\sqrt{6}}{4}\right) d+4 \\
& +\frac{\sqrt{15}}{3}-\frac{15 \sqrt{6}}{4} \\
& G A_{e}\left(G B G\left(C_{m}, P_{3} ; d\right)\right)=d(m-3)\left(\frac{2 \sqrt{2 \times 2}}{2+2}\right)+4\left(\frac{2 \sqrt{2 \times 3}}{2+3}\right) \\
& +2(d-2)\left(\frac{2 \sqrt{2 \times 4}}{2+4}\right)+6\left(\frac{2 \sqrt{3 \times 3}}{3+3}\right) \\
& +2\left(\frac{2 \sqrt{3 \times 4}}{3+4}\right)+(7 d-15)\left(\frac{2 \sqrt{4 \times 4}}{4+4}\right) \\
& =d(m-3)+\left(\frac{4 \sqrt{2}}{3}+7\right) d+\frac{8 \sqrt{6}}{5}+6 \\
& +\frac{8 \sqrt{3}}{7}-\frac{8 \sqrt{2}}{3}-15 \text {. }
\end{aligned}
$$

The proof is complete. $\square$
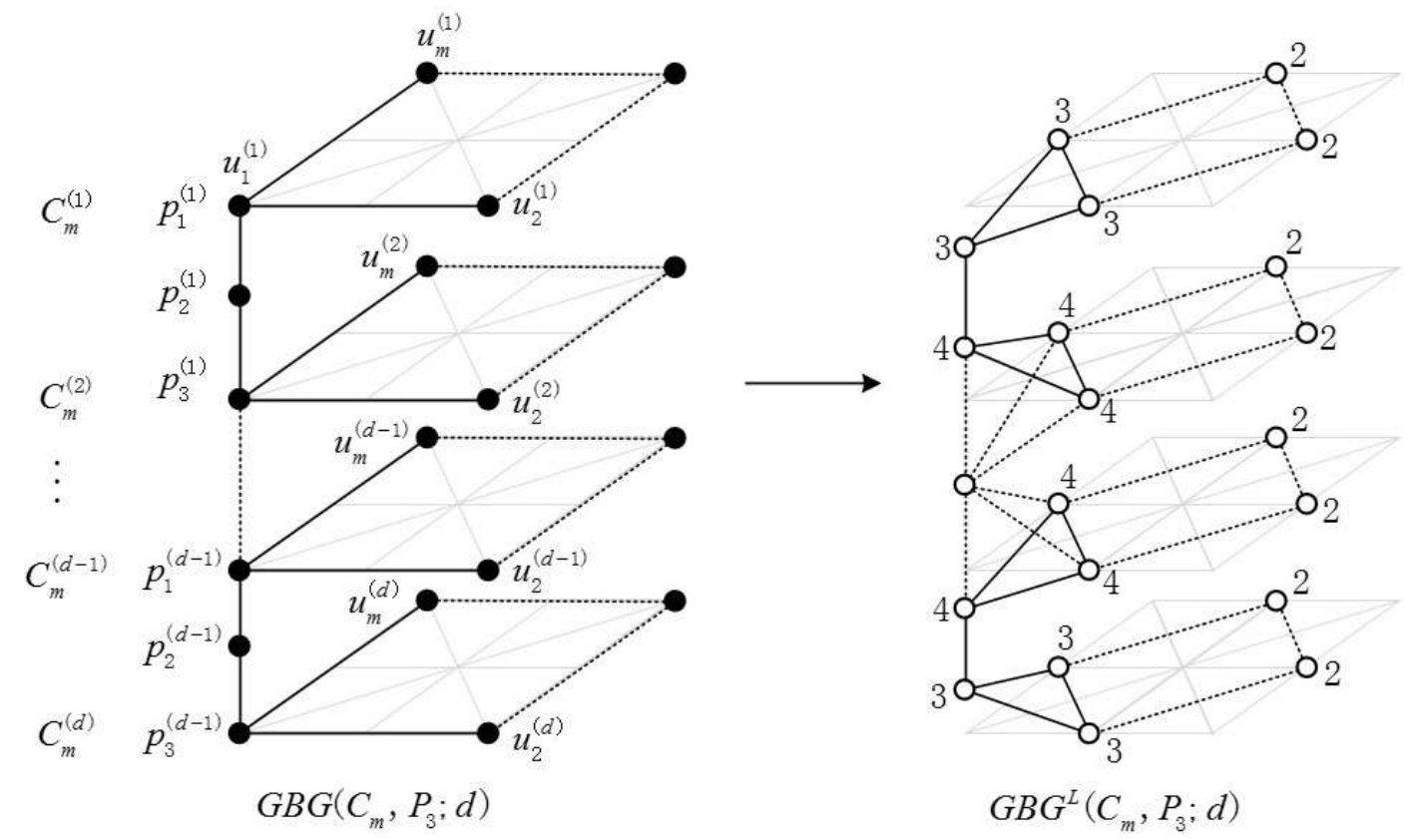

Figure 11. The generalized bridge molecular graph of $G B G\left(C_{m}, P_{3} ; d\right)$ and $G B G^{L}\left(C_{m}, P_{3} ; d\right)$.

For Example 5, in Figure 12, (cyclohexane-1,1-diylbis(methylene))dicyclohexane can be modeled by $G B G\left(C_{6}, P_{3} ; 3\right)$, so $A B C_{e}\left(G B G\left(C_{6}, P_{3} ; 3\right)\right) \approx 19.57183078$ and $G A_{e}\left(G B G\left(C_{6}, P_{3} ; 3\right)\right) \approx 28.78428831$. 


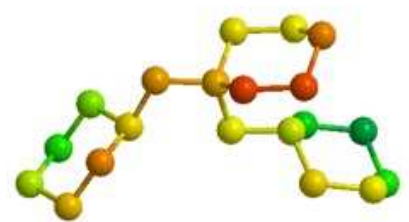

(a)

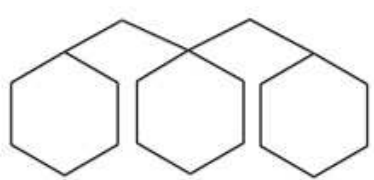

(b)

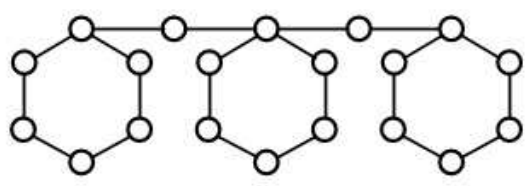

(c)

Figure 12. (a) (cyclohexane-1,1-diylbis(methylene))dicyclohexane ball and stick model graph in $3 D$; (b) (cyclohexane-1,1-diylbis(methylene))dicyclohexane chemical structure graph; and (c) (cyclohexane-1,1-diylbis(methylene)) dicyclohexane model graph in chemical graph theory.

Theorem 6. Let $G B G\left(C_{m}, P_{2} ; d\right)$ be the generalized bridge molecular graph for $n=2, d \geq 4$, and $m \geq 3$ (see Figure 13), then the $A B C_{e}$ and $G A_{e}$ of $G B G\left(C_{m}, P_{2} ; d\right)$ are

$$
\begin{aligned}
A B C_{e}\left(G B G\left(C_{m}, P_{2} ; d\right)\right)= & \frac{\sqrt{2}}{2} d m+\left(\sqrt{2}+\frac{\sqrt{6}}{4}+\frac{4 \sqrt{3}}{3}+\frac{\sqrt{10}}{6}-\frac{3 \sqrt{2}}{2}\right) d \\
& +\frac{4}{3}+4 \sqrt{\frac{2}{5}}+2 \sqrt{\frac{7}{5}}+2 \sqrt{\frac{3}{10}}-\frac{\sqrt{6}}{2}-4 \sqrt{3}-\frac{2 \sqrt{10}}{3}, \\
G A_{e}\left(G B G\left(C_{m}, P_{2} ; d\right)\right)= & d m+\left(\frac{4 \sqrt{2}}{3}+\frac{8 \sqrt{6}}{5}-1\right) d+\sqrt{15}+\frac{16 \sqrt{5}}{9}+\frac{4 \sqrt{30}}{11} \\
& -\frac{16 \sqrt{6}}{5}-\frac{8 \sqrt{2}}{3}-4 .
\end{aligned}
$$

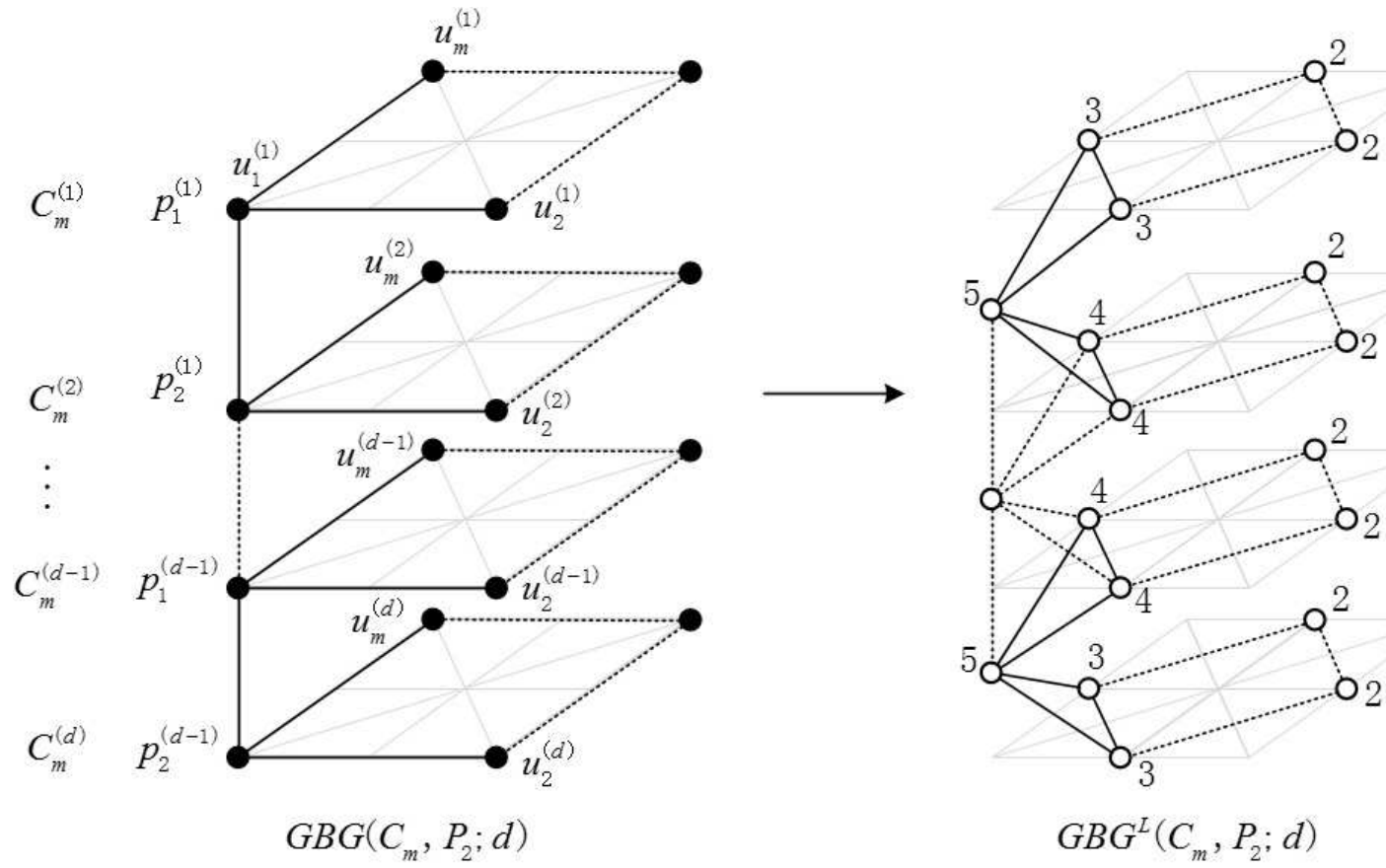

Figure 13. The generalized bridge molecular graph of $G B G\left(C_{m}, P_{2} ; d\right)$ and $G B G^{L}\left(C_{m}, P_{2} ; d\right)$.

Proof. In Figure 13, the degrees of vertices in line graph $G^{L}\left(G B G\left(C_{m}, P_{2} ; d\right)\right)$ are displayed near by the corresponding vertices. This line graph has $d(m-5)-6$ edges. In addition, there are $d(m-3)$ edges of type $d_{L(G)}\left(e_{1}\right)=d_{L(G)}\left(e_{2}\right)=2,4$ edges of type $d_{L(G)}\left(e_{1}\right)=2, d_{L(G)}\left(e_{2}\right)=3,2(d-2)$ edges of type $d_{L(G)}\left(e_{1}\right)=2, d_{L(G)}\left(e_{2}\right)=4,2$ edges of type $d_{L(G)}\left(e_{1}\right)=d_{L(G)}\left(e_{2}\right)=3,4$ edges of type $d_{L(G)}\left(e_{1}\right)=3, d_{L(G)}\left(e_{2}\right)=5, d-2$ edges of type $d_{L(G)}\left(e_{1}\right)=d_{L(G)}\left(e_{2}\right)=4,4$ edges of type 
$d_{L(G)}\left(e_{1}\right)=4, d_{L(G)}\left(e_{2}\right)=5,4(d-3)$ edges of type $d_{L(G)}\left(e_{1}\right)=4, d_{L(G)}\left(e_{2}\right)=6,2$ edges of type $d_{L(G)}\left(e_{1}\right)=5, d_{L(G)}\left(e_{2}\right)=6$, and $d-4$ edges of type $d_{L(G)}\left(e_{1}\right)=d_{L(G)}\left(e_{2}\right)=6$. Hence, we have

$$
\begin{aligned}
& A B C_{e}\left(G B G\left(C_{m}, P_{2} ; d\right)\right)=d(m-3)\left(\sqrt{\frac{2+2-2}{2 \times 2}}\right)+4\left(\sqrt{\frac{2+3-2}{2 \times 3}}\right) \\
& +2(d-2)\left(\sqrt{\frac{2+4-2}{2 \times 4}}\right)+2\left(\sqrt{\frac{3+3-2}{3 \times 3}}\right) \\
& +4\left(\sqrt{\frac{3+5-2}{3 \times 5}}\right)+(d-2)\left(\sqrt{\frac{4+4-2}{4 \times 4}}\right) \\
& +4\left(\sqrt{\frac{4+5-2}{4 \times 5}}\right)+4(d-3)\left(\sqrt{\frac{4+6-2}{4 \times 6}}\right) \\
& +2\left(\sqrt{\frac{5+6-2}{5 \times 6}}\right)+(d-4)\left(\sqrt{\frac{6+6-2}{6 \times 6}}\right) \\
& =\frac{\sqrt{2}}{2} d m+\left(\sqrt{2}+\frac{\sqrt{6}}{4}+\frac{4 \sqrt{3}}{3}+\frac{\sqrt{10}}{6}-\frac{3 \sqrt{2}}{2}\right) d \\
& +\frac{4}{3}+4 \sqrt{\frac{2}{5}}+2 \sqrt{\frac{7}{5}}+2 \sqrt{\frac{3}{10}}-\frac{\sqrt{6}}{2}-4 \sqrt{3}-\frac{2 \sqrt{10}}{3}, \\
& G A_{e}\left(G B G\left(C_{m}, P_{2} ; d\right)\right)=d(m-3)\left(\frac{2 \sqrt{2 \times 2}}{2+2}\right)+4\left(\frac{2 \sqrt{2 \times 3}}{2+3}\right) \\
& +2(d-2)\left(\frac{2 \sqrt{2 \times 4}}{2+4}\right)+2\left(\frac{2 \sqrt{3 \times 3}}{3+3}\right) \\
& +4\left(\frac{2 \sqrt{3 \times 5}}{3+5}\right)+(d-2)\left(\frac{2 \sqrt{4 \times 4}}{4+4}\right) \\
& +4\left(\frac{2 \sqrt{4 \times 5}}{4+5}\right)+4(d-3)\left(\frac{2 \sqrt{4 \times 6}}{4+6}\right) \\
& +2\left(\frac{2 \sqrt{5 \times 6}}{5+6}\right)+(d-4)\left(\frac{2 \sqrt{6 \times 6}}{6+6}\right) \\
& =d m+\left(\frac{4 \sqrt{2}}{3}+\frac{8 \sqrt{6}}{5}-1\right) d+\sqrt{15}+\frac{16 \sqrt{5}}{9}+\frac{4 \sqrt{30}}{11} \\
& -\frac{16 \sqrt{6}}{5}-\frac{8 \sqrt{2}}{3}-4
\end{aligned}
$$

The proof is complete. $\gamma_{i}\left(C_{6} \square C_{n}\right)$

For Example 6, in Figure 14, 2'H, $2^{\prime \prime} H-1,1^{\prime}: 1^{\prime}, 1^{\prime \prime}: 1^{\prime \prime}, 1^{\prime \prime \prime}$-quaterphenyl can be modeled by $G B G\left(C_{6}, P_{2} ; 4\right)$, so $A B C_{e}\left(G B G\left(C_{6}, P_{2} ; 4\right)\right) \approx 25.00131406$ and $G A_{e}\left(G B G\left(C_{6}, P_{2} ; 4\right)\right) \approx 37.44953704$.

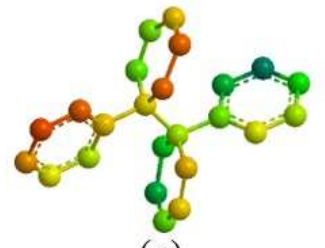

(a)

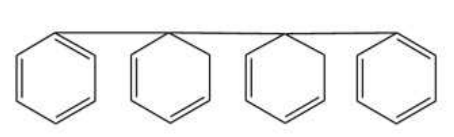

(b)

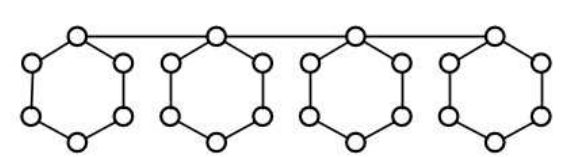

(c)

Figure 14. (a) $2^{\prime} H, 2^{\prime \prime} H-1,1^{\prime}: 1^{\prime}, 1^{\prime \prime}: 1^{\prime \prime}, 1^{\prime \prime \prime}$-quaterphenyl ball and stick model graph in $3 D$; (b) $2^{\prime} H, 2^{\prime \prime} H-1,1^{\prime}: 1^{\prime}, 1^{\prime \prime}: 1^{\prime \prime}, 1^{\prime \prime \prime}$-quaterphenyl chemical structure graph; and (c) $2^{\prime} H, 2^{\prime \prime} H-1,1^{\prime}: 1^{\prime}, 1^{\prime \prime}$ : $1^{\prime \prime}, 1^{\prime \prime \prime}$-quaterphenyl model graph in chemical graph theory. 


\section{Conclusions}

Topological indices are proven to be very helpful to test the chemical properties of new chemical or physical materials. To describe more kinds of long chain polymerization products than the bridge molecular graphs, we propose the generalized bridge molecular graph structures. In this paper, we focus on some generalized bridge molecular graphs such as $G B G\left(S_{m}, P_{n} ; d\right)$ and $G B G\left(C_{m}, P_{n} ; d\right)$ and give the formulas of the edge version $A B C$ and $G A$ indices of these generalized bridge molecular graphs. By demonstrating the calculation of real molecules, we find that some long chain molecular graphs can be quickly modeled and their topological indices can be calculated using generalized bridge molecular graphs. The results of this paper also offer promising prospects in the applications for chemical and material engineering, especially in chemical industry research.

Author Contributions: X.Z. contribute for conceptualization, designing the experiments, wrote original draft preparation and funding; J.-B.L. contribute for supervision, methodology, project administration and formal analysing; X.W. contribute for performed experiments, validation. M.K.J. for resources, software, some computations; S.A. reviewed and edited initial draft and wrote the final draft; M.R.F. analyzed the data curation; All authors read and approved the final version of the paper.

Funding: This work was supported by the key project of the Sichuan Provincial Department of Education under Grant No. 17ZA0079 and 18ZA0118, partially supported by China Postdoctoral Science Foundation under Grant No. 2017M621579 and Postdoctoral Science Foundation of Jiangsu Province under Grant No. 1701081B, Project of Anhui Jianzhu University under Grant No. 2016QD116 and 2017dc03, and the Soft Scientific Research Foundation of Sichuan Provincial Science and Technology Department under Grant No. 2018ZR0265.

Acknowledgments: The authors are grateful to the anonymous reviewers and the editor for the valuable comments and suggestions.

Conflicts of Interest: The authors declare no conflict of interest.

\section{References}

1. Harary, F.; Norman, R.Z. Some properties of line digraphs. Rendiconti del Circolo Matematico di Palermo 1960, 9, 161-169. [CrossRef]

2. Wiener, H. Structural determination of paraffin boiling points. J. Am. Chem. Soc. 1947, 69, 7-20. [CrossRef]

3. Iranmanesh, A.; Gutman, I.; Khormali, O.; Mahmiani, A. The edge versions of the wiener index. MATCH Commun. Math. Comput. Chem. 2009, 61, 663-672.

4. Devillers, J.; Balaban, A.T. Topological Indices and Related Descriptors in QSAR and QSPR; CRC Press: Boca Raton, FL, USA, 1999.

5. Gao, W.; Farahani, M.R.; Wang, S.H.; Husin, M.N. On the edge-version atom-bond connectivity and geometric arithmetic indices of certain graph operations. Appl. Math. Comput. 2017, 308, 11-17. [CrossRef]

6. Gao, W.; Siddiqui, M.K.; Imran, M.; Jamil, M.K.; Farahani, M. R. Forgotten topological index of chemical structure in drugs. Saudi Pharm. J. 2016, 24, 258. [CrossRef] [PubMed]

7. Nikolić, S.; Kovačević, G.; Miličević, A.; Trinajstić, N. The Zagreb indices 30 years after. Croat. Chem. Acta 2003, 76, 113-124.

8. Estrada, E.; Torres, L.; Rodriguez, L.; Gutman, I. An atom-bond connectivity index: Modelling the enthalpy of formation of Alkanes. Indian J. Chem. 1998, 37A, 849-855.

9. Dimitrov, D. Efficient computation of trees with minimal atom-bond connectivity index. Appl. Math. Comput. 2013, 224, 663-670. [CrossRef]

10. Shao, Z.; Wu, P.; Gao, Y.; Gutman, I.; Zhang, X.J. On the maximum ABC index of graphs without pendent vertices. Appl. Math. Comput. 2017, 315, 298-312. [CrossRef]

11. Shao, Z.; Wu, P.; Zhang, X.; Dimitrov, D.; Liu, J.B. On the maximum ABC index of graphs with prescribed size and without pendent vertices. IEEE Access 2018, 6, 27604-27616. [CrossRef]

12. Farahani, M.R. The edge version of atom bond connectivity index of connected graph. Acta Univ. Apul. 2012, 36, 277-284.

13. Gao, W.; Husin, M.N.; Farahani, M.R.; Imran, M. On the Edges Version of Atom-Bond Connectivity Index of Nanotubes. J. Comput. Theor. Nanosci. 2017, 13, 6733-6740. [CrossRef]

14. Farahani, M.R. The Edge Version of Geometric-Arithmetic Index of Benzenoid Graph. Proc. Romanian Acad. Ser. B 2013, 15, 95-98. 
15. Vukicevic, D.; Furtula, B. Topological index based on the ratios of geometric and arithmetical means of end-vertex degrees of edges. J. Math. Chem. 2009, 46, 1369-1376. [CrossRef]

16. Martińez-Peŕez, A.; Rodriǵuez, J.M,; Sigarreta, J.M. CMMSE: A new approximation to the geometric-arithmetic index. J. Math. Chem. 2017, 4, 1-19.

17. Baig, A.Q.; Imran, M.; Khalid, W.; Naeem, M. Molecular description of carbon graphite and crystal cubic carbon structures. Can. J. Chem. 2017, 95, 674-686. [CrossRef]

18. Gutman, I.; Furtula, B.; Das, K.C. Extended energy and its dependence on molecular structure. Can. J. Chem. 2017, 95, 526-529. [CrossRef]

19. Mahmiani, A.; Khormali, O.; Iranmanesh, A. On the edge version of geometric-arithmetic index. Dig. J. Nanomater. Biostruct. 2012, 7, 411-414.

20. Zafar, S.; Nadeem, M.F.; Zahid, Z. On the edge version of geometric-arithmetic index of nanocones. Stud. Univ. Babes-Bolyai Chem. 2016, 61, 273-282.

21. Gao, W.; Husin, M.N.; Farahani,M.R.; Imran, M. On the Edges Version of Atom-Bond Connectivity and Geometric Arithmetic Indices of Nanocones CNCk. J. Comput. Theor. Nanosci. 2016, 13, 6741-6746. [CrossRef]

(C) 2018 by the authors. Licensee MDPI, Basel, Switzerland. This article is an open access article distributed under the terms and conditions of the Creative Commons Attribution (CC BY) license (http://creativecommons.org/licenses/by/4.0/). 